\title{
Detección de zonas de fracturamiento en áreas urbanas mediante el método capacitivo de tomografía eléctrica
}

\author{
Ana Belén Olvera-Ramírez* \\ René E. Chávez* \\ Juan Esteban Hernández-Quintero*
}

Recibido en febrero de 2014; aceptado en octubre de 2014

\begin{abstract}
Within the metropolitan area of Mexico City; affectations are commonly observed in buildings structures, and in some cases fractures, subsidence and collapses have become one of the major risks in the current urban sprawl. Iztacalco municipality is located northeastward of the city. Surrounding municipalities are Venustiano Carranza and Cuauhtémoc to the north; Benito Juárez to the west; Iztapalapa to south and Nezahualcóyotl to east. Several areas are influenced of disruption in their housing units, such as Barrio de Santiago and "Infonavit" (Institute of the National Housing Fund for Workers). Damage to the units are related to the presence of fractures in the subsurface under structures, weakening or altering their basements underground and causing structural damage. In order to characterize the observed fracturing, geophysical surveys were carried out in two critical areas of these residential buildings; consisting of four surveys of Electrical Capacitive Tomography (ECT) in each site. A preferential NW-SE direction of fractures inferred in the subsurface in both study areas were found. Such directions have a remarkable correlation with previous studies near this area.

In the same manner, the acquired data using the ECT method with the Omh Mapper (Geometrics) instrument, are noisy because the shallow variations of electrical resistivity; in order to minimize such noise, the data were processed and inverted separately and display clear the resistivity anomalies observed in the profiles.

Key words: Electrical Resistivity Tomography, subsidence, fractures.
\end{abstract}

* Instituto de Geofísica, Universidad Nacional Autónoma de México, Ciudad Universitaria, Circuito de la Investigación Científica, 04510 México, D.F. 


\section{Resumen}

Dentro de la zona metropolitana de la Ciudad de México; es común observar afectaciones de estructuras en los edificios, y en algunos casos fracturas, hundimientos y colapsos se han convertido en uno de los principales riesgos en las manchas urbanas actuales. La delegación Iztacalco, se encuentra en al centro-oriente del Distrito Federal, limita al norte con la delegación Venustiano Carranza y Cuauhtémoc, al poniente con Benito Juárez, al sur con Iztapalapa y al oriente con el municipio mexiquense de Nezahualcóyotl, presenta en algunas zonas, afectaciones en sus unidades habitacionales, ejemplos claros son el Barrio de Santiago y la Unidad Habitacional "Infonavit" (Instituto del Fondo Nacional de la Vivienda para los Trabajadores). Los daños a las unidades se deben a la existencia de fracturas en el subsuelo que pasan por debajo de las estructuras civiles, debilitando sus cimentos o alterando el subsuelo, originando los daños estructurales que afectan estas construcciones. Para caracterizar el fracturamiento observado, se llevaron a cabo estudios geofísicos en las dos unidades habitacionales, donde se realizaron cuatro perfiles eléctricos para cada una de las unidades, mediante el método de Tomografía Eléctrica Capacitiva (TEC). Se encontró una dirección preferencial de las fracturas inferidas en el subsuelo en ambas zonas de estudio con dirección NW-SE. Esta dirección se logra correlacionar con la orientación de fracturas inferidas descritas en estudios previamente realizados en la delegación Iztacalco, que se encuentran muy cercanos a las zonas de estudio.

De igual forma los datos obtenidos mediante el método capacitivo con el instrumento Ohm-Mapper, se ven afectados por ruido cuyo origen, en su mayoría, se debe a variaciones superficiales de la resistividad, dichos datos fueron procesados de manera separada, para minimizar el efecto de ruido, y lograr visualizar de forma más clara las anomalías expuestas en los perfiles.

Palabras clave: Tomografia de Resistividad Eléctrica, subsidencia, fracturas.

\section{Introducción}

Las zonas con mayor vulnerabilidad a fracturamientos y efectos de subsidencia diferencial en el subsuelo deben de ser identificadas empleando diferentes técnicas geofísicas para prevenir accidentes y eventos desastrosos, que representan un alto costo socio-económico para las autoridades responsables. Hoy en día, los métodos geofísicos se han convertido en una herramienta muy importante para caracterizar el subsuelo donde se encuentra la infraestructura urbana. Estas metodologías permiten cuantificar las propiedades del terreno para definir las características físicas del subsuelo, antes y después de la construcción de obras públicas y privadas, así como sus edificaciones. 
Los métodos de prospección utilizados en la exploración del subsuelo enfrentan un importante desafío, cuando se emplean en zonas fuertemente urbanizadas como: casas, museos, escuelas, monumentos históricos, etc., representan un obstáculo para la adecuada adquisición de la información geofísica. En la mayoría de los casos, estas construcciones son el objeto de estudio, y es necesario caracterizar el subsuelo sobre el que se encuentran estas, es decir que estas edificaciones impiden el diseño de los perfiles geofísicos de forma paralela para formar redes que permitan una visualización correcta del problema a profundidad. Además, no es posible de igual forma barrenar el suelo para la colocación de detectores, debido a la cantidad de cables de luz y teléfono, tuberías de gas o drenaje, que podrían ser dañados con el estudio (Chávez et al., 2014).

Por lo tanto, el objetivo principal de caracterizar el subsuelo, para detectar la anomalías que puedan afectar la estabilidad de las construcciones urbanas, principalmente cuando estos rasgos no pueden ser seguidas a través de diferentes obstáculos representados por construcciones $u$ otro tipo de problemas de origen natural o creado por el hombre (Trogu et al., 2011), conlleva al empleo de equipos y técnicas especiales que permitan de forma adecuada el reconocimiento a profundidad de este tipo de zonas.

Hoy en día se cuenta con equipos y técnicas especiales para llevar a cabo estudios geofísicos en zonas urbanas que son de gran ayuda para el reconocimiento del subsuelo y con una forma de adquisición de datos que evita dañar la zona de estudio.

Este método se le denomina Tomografía Eléctrica Capacitiva (TEC), la cual nos proporciona una interpretación del comportamiento de la resistividad eléctrica en el subsuelo, mediante la adquisición de datos con el instrumento denominado OhmMapper (Geometrics Inc., Estados Unidos).

La compleja geología de la cuenca de México, ha proporcionado a lo largo de la historia abundantes recursos de agua a sus habitantes, sin embargo, la escasez de agua superficial ha propiciado la sobreexplotación de acuíferos profundos, lo que ha provocado problemas de inestabilidad en el subsuelo. Estos problemas están asociados con aspectos relacionados con la composición geológica-estructural de la misma, debido a la distribución de los materiales geológicos que la forman.

El desarrollo urbano en las ciudades modernas requiere de un conocimiento más integral del subsuelo, sobre todo en aquellas áreas, donde aumentan las concentraciones humanas. Tomando en cuenta la inadecuada forma de planeación del crecimiento poblacional y la falta de concientización acerca del uso de los recursos naturales, se ha provocado, en términos de la explotación de los acuíferos subterráneos, el descenso continuo del nivel de estos.

El presente trabajo, trata dos ejemplos en donde el problema de la sobreexplotación de los acuíferos y el pobre mantenimiento de la infraestructura hidráulica ha afectado la infraestructura urbana. 
Se caracteriza el subsuelo de dos zonas habitacionales, un edificio perteneciente a la Unidad Habitacional Infonavit y otra al Barrio de Santiago, en la delegación Iztacalco. En ambas zonas se describen los estudios geofísicos realizados con un equipo de resistividad que utiliza el método capacitivo. Las fracturas que afectan estos sectores, se definen de forma razonable y explican el deterioro de las construcciones.

\section{Método capacitivo}

La aplicación geofísica de la técnica eléctrica con acoplamiento capacitivo se desarrolla a partir de la investigación realizada en la década de los cincuenta, por el Departamento de Defensa de Estados Unidos, donde el requisito era detectar grietas ocultas en Groenlandia y la Antártida. En la década de los setenta, el trabajo pionero fue llevado a cabo en Rusia por Timofeev (Kuras et al., 2005).

Es una técnica geofísica emergente diseñada para extender el alcance de los métodos convencionales de resistividad de corriente continua. La técnica se basa en la naturaleza capacitiva del mecanismo de acoplamiento entre los sensores y el suelo, en contraste con otras técnicas de resistividad que se basan en el acoplamiento galvánico. La resistividad capacitiva se basa en el principio de medición bien conocida de resistividad convencional salvo que los electrodos galvánicos se sustituyen por sensores capacitivos.

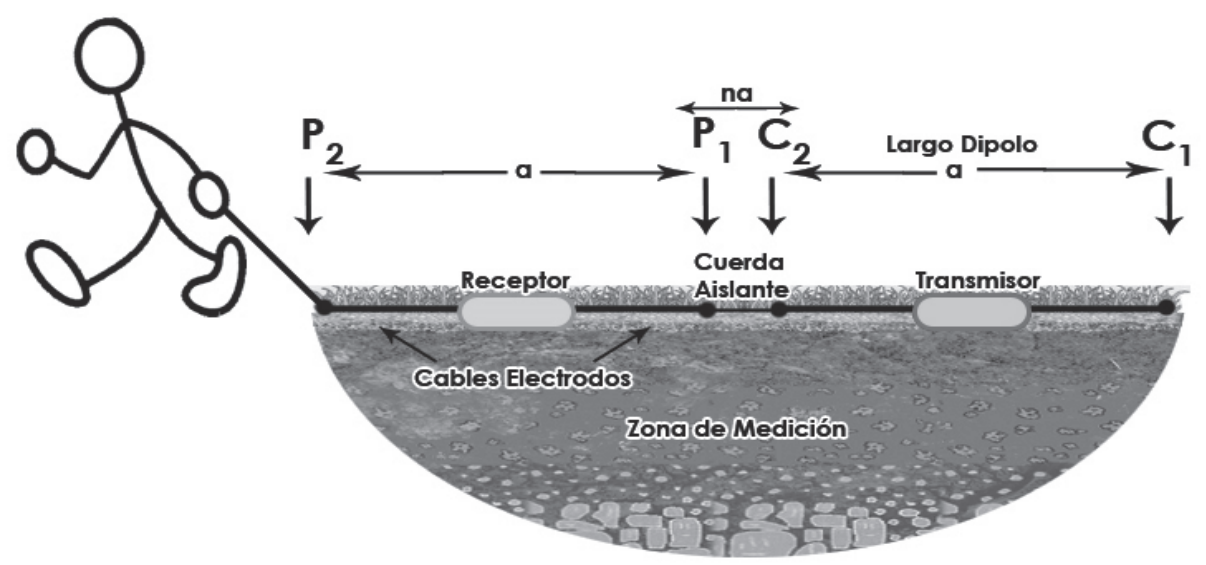

Figura 1. Representación del arreglo dipolo-dipolo en la resistividad capacitiva. Este método consiste en utilizar dos electrodos, $\mathrm{C} 1$ y $\mathrm{C} 2$, ubicados en la superficie y conectados a una fuente de poder y la medida de diferencias de potencial entre puntos se mide con dos electrodos de potencial, P1 y P2. 
El método se basa en un arreglo simple de cable coaxial con secciones de transmisor y receptor, que son deslizadas a lo largo del terreno. Una corriente es acoplada a la tierra por el transmisor y medida en el receptor, el cual basa su funcionamiento en el arreglo dipolo-dipolo, (Figura 1). Este tipo de arreglo consiste en cuatro electrodos dispuestos en forma lineal siguiendo la secuencia A B M N, el espacio entre los electrodos de corriente A B se denomina factor $a$, que será la misma distancia entre los electrodos de potencial M y N. Se debe hacer que el factor $a$ se mantenga fijo y el factor $n$ se incremente.

La profundidad a la cual los datos del Ohm-Mapper pueden ser interpretados confiablemente, depende del tamaño del dipolo y la distancia entre el dipolo transmisor y el dipolo receptor. La distancia adecuada para que el receptor pueda localizar al transmisor depende de la resistividad del subsuelo.

La expresión para el cálculo de la resistividad con el arreglo dipolo-dipolo es:

$$
\rho_{a}=2 \pi a \frac{V}{I}\left\{\frac{1}{\frac{1}{n}-\frac{2}{n+1}+\frac{1}{n+2}}\right\}
$$

Donde $a$ es el espacio entre dipolos, y $n$ los múltiplos enteros de $a$. Este método presenta buena resolución para los contrastes laterales de resistividad, define mejor los valores de resistividad aparente en niveles cercanos a la superficie, dado que conforme se incrementan los niveles a profundidad habrá pérdida de señal. Este tipo de arreglo es altamente susceptible a los cambios horizontales en la resistividad del medio y menos eficiente para detectar cambios verticales de resistividad, es mejor para encontrar estructuras verticales como diques y cavidades pero deficiente para estructuras estratificadas (Chávez et al., 2009, 2010).

\section{Bosquejo geológico de la zona de estudio}

El significado de Iztacalco viene de las palabras en náhuatl, que por su toponimia y relación con términos de geotecnia e ingeniería civil se define como: "lugar de las casas blancas o casas de la sal", de Iztatl, sal, calli, casa, y co, lugar. Esto sugiere el tipo de terrenos en donde se encontraba asentado este lugar.

Esta zona de pequeñas islas, pantanos y cañaverales, fue transformada en fértiles campos de cultivo, mediante el avanzado sistema de chinampas. A la llegada de los españoles Iztacalco era uno de los muchos calpullis de Tenochtitlán conformado por diversos barrios. 
Las zonas que se estudian en este artículo, se ubican en la delegación Iztacalco, la cual se localiza hacia la porción centro-oriente del Distrito Federal. Limita al norte con las delegaciones Venustiano Carranza y Cuauhtemoc, al poniente con la delegación Benito Juárez, al sur con la delegación Iztapalapa y al oriente con el municipio mexiquense de Nezahualcóyotl. Es la delegación más pequeña de 16 delegaciones que comparten el territorio capitalino, con apenas $23.3 \mathrm{~km}^{2}$, y alberga una población cercana a los 400 mil habitantes.

\section{Unidad Habitacional Infonavit}

La primera zona de estudio es un complejo habitacional perteneciente a la Unidad Habitacional Infonavit (Instituto del Fondo Nacional de la Vivienda para los Trabajadores), se localiza en la porción centro-suroeste de la delegación Iztacalco. La construcción de este conjunto habitacional se realizó con la finalidad de crear un proyecto de viviendas para los trabajadores, que nació durante el gobierno del presidente Adolfo López Mateos en 1962. Esta unidad ocupó una superficie de 35ha, está conformada por 5,130 viviendas con una población cercana a las 23,000 personas. En esta unidad se podían encontrar en su inauguración instalaciones de servicio como un edificio social, zonas comerciales, centros deportivos, planteles educativos y un lago artificial de $6,000 \mathrm{~m}^{2}$ de superficie (Figura 2).

Desafortunadamente, el mencionado lago desapareció a consecuencia de una fractura provocada por el sismo de 1985, que afectó varios edificios aledaños. Por tal motivo, se construyó un parque en el 2004 sobre el ex lago de la unidad. Sin embargo, y a pesar de esta remediación, han aparecido nuevas fracturas ocasionando fuertes daños en las construcciones, además de una serie de hundimientos en algunos de los edificios, que son parte de este conjunto.

El complejo habitacional consta de cuatro edificios de cinco pisos cada uno. La Dirección de Protección Civil de la delegación Iztacalco señala que esta estructura presenta fuertes daños en su base, fisuras y grietas significativas, además de una separación importante entre los edificios. Para el estudio del área afectada se realizaron cuatro perfiles (Figura 3), en donde se intentó rodear un edificio que presenta hundimientos y fisuras en su estructura, efectuando el levantamiento con cinco receptores, la longitud total de los perfiles fue de 380 metros.

A partir de los datos obtenidos se generaron cuatro modelos bidimensionales, los cuales corresponden a cada una de las líneas de estudio. En esta zona se pueden percibir a simple vista una amplia serie de rellenos de concreto, tanto cerca como lejos de las tuberías sanitarias (atarjeas), también es apreciable que el suelo se encuentra fracturado, de igual manera es posible observar una serie de hundimientos en la parte baja de los edificios (Figuras 4 y 5). Se pretende inferir la distribución de 


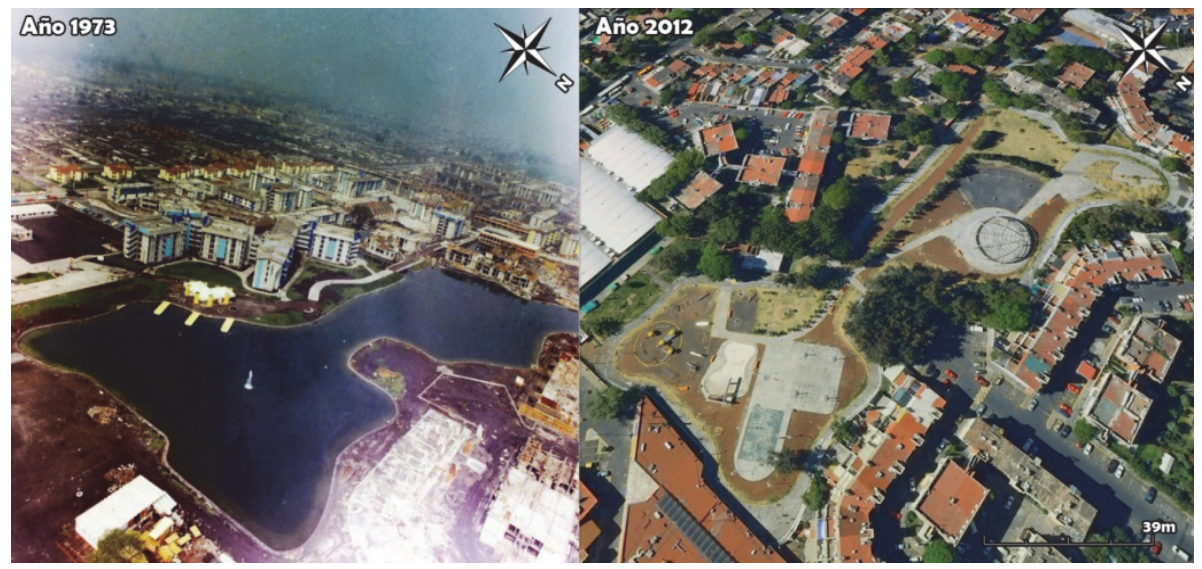

Figura 2. Comparación en vista aérea de la Unidad Habitacional Infonavit Iztacalco, mostrando el lago en el año de 1973 y su vista actual.

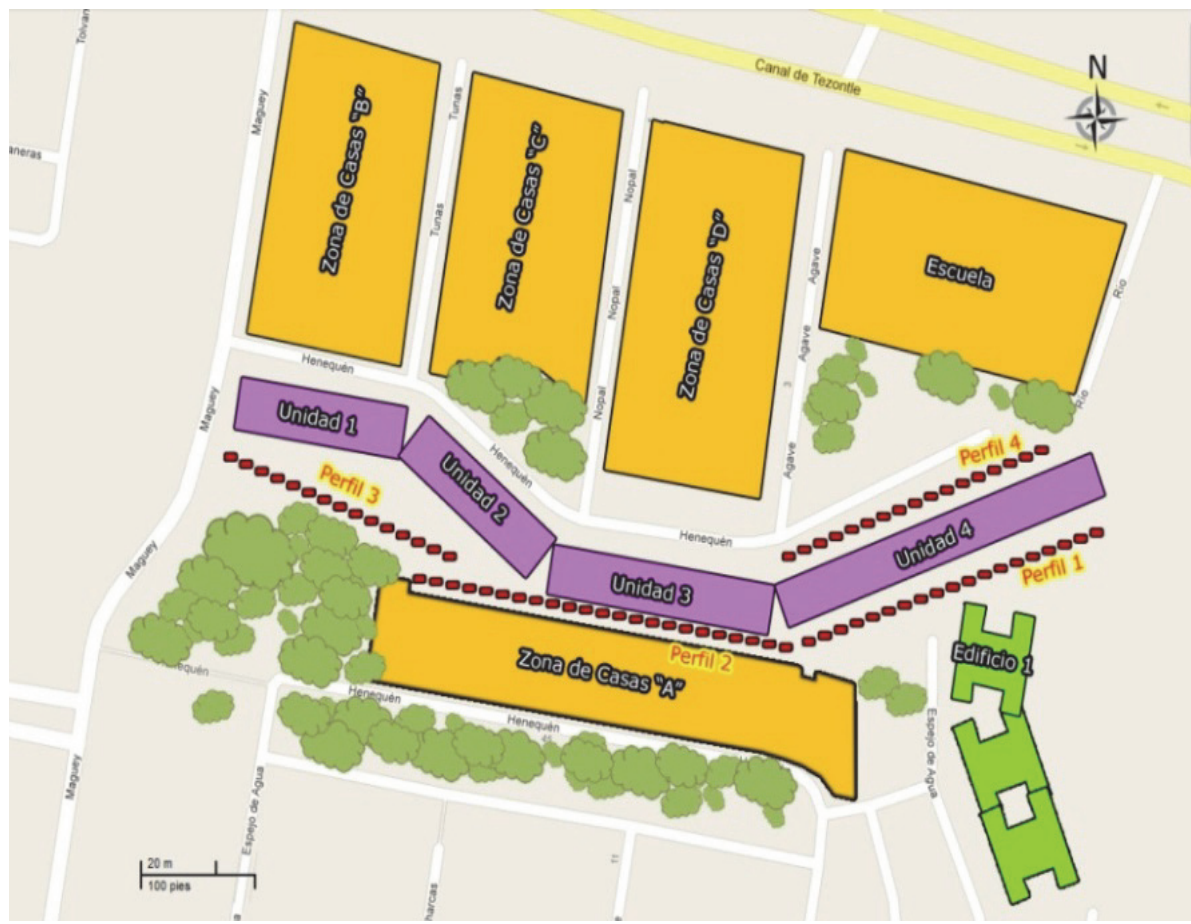

Figura 3. Localización de los perfiles en el area correspondiente a la Unidad Habitacional Infonavit. 

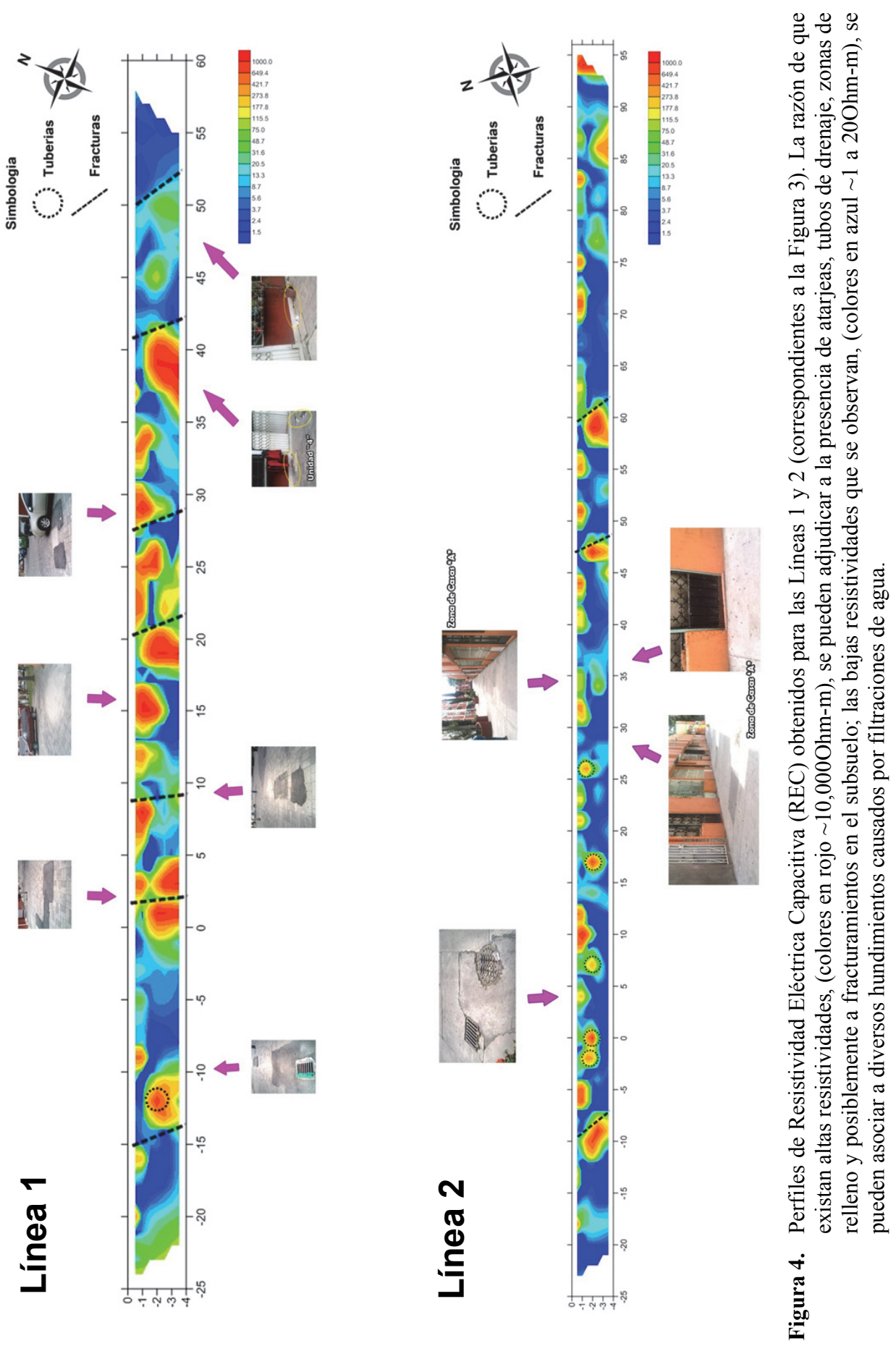


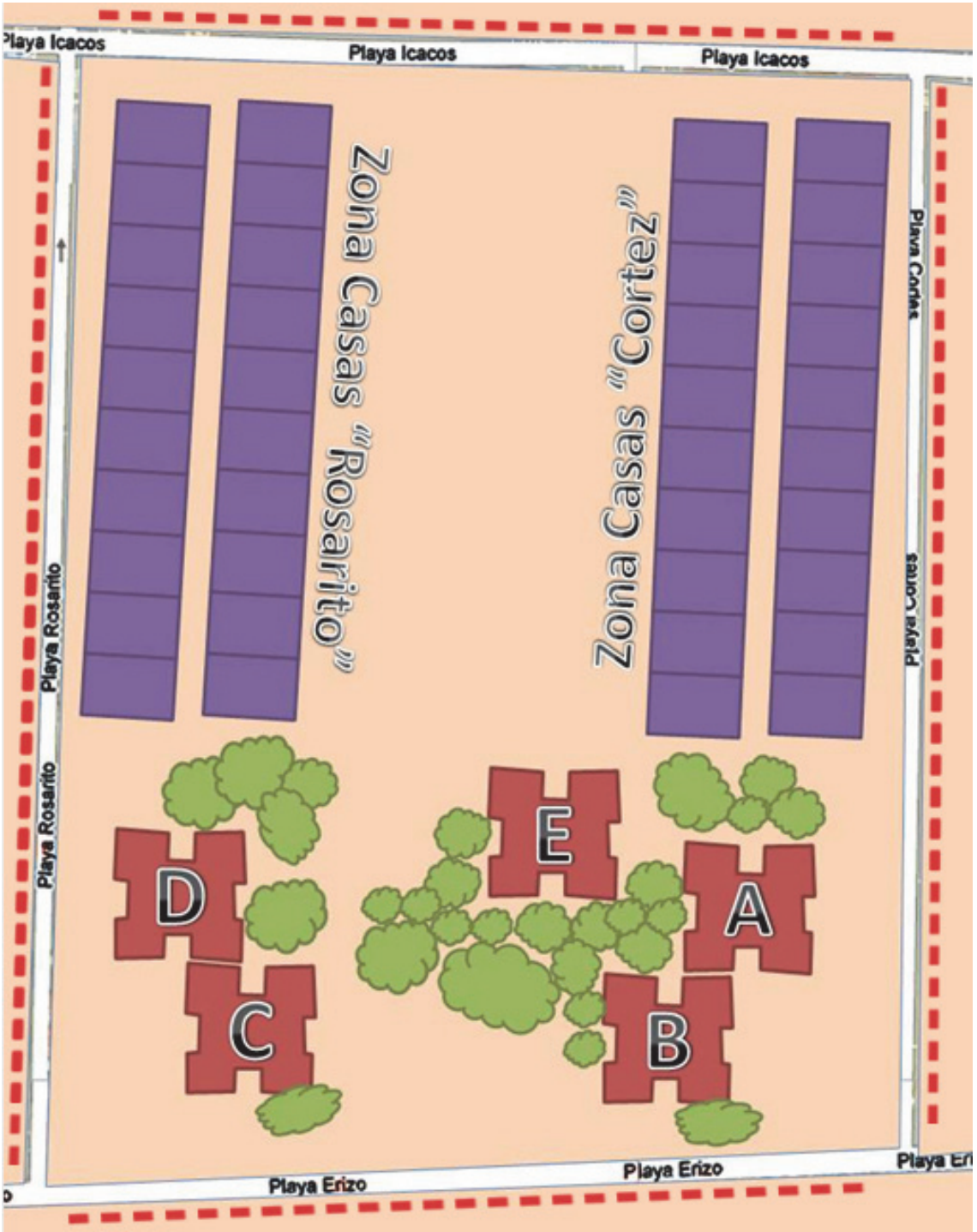

Figura 5. Localización de los perfiles en la zona de casas, correspondiente al Barrio de Santiago.

las fracturas en la zona de estudio, mediante los contrastes que representan cambios de material o discontinuidades en el medio, los valores de alta resistividad y las bajas resistividades que se observan, se pueden asociar a diversos hundimientos causados por filtraciones de agua o por altas concentraciones de este fluido en las arcillas que soportan al complejo habitacional. 


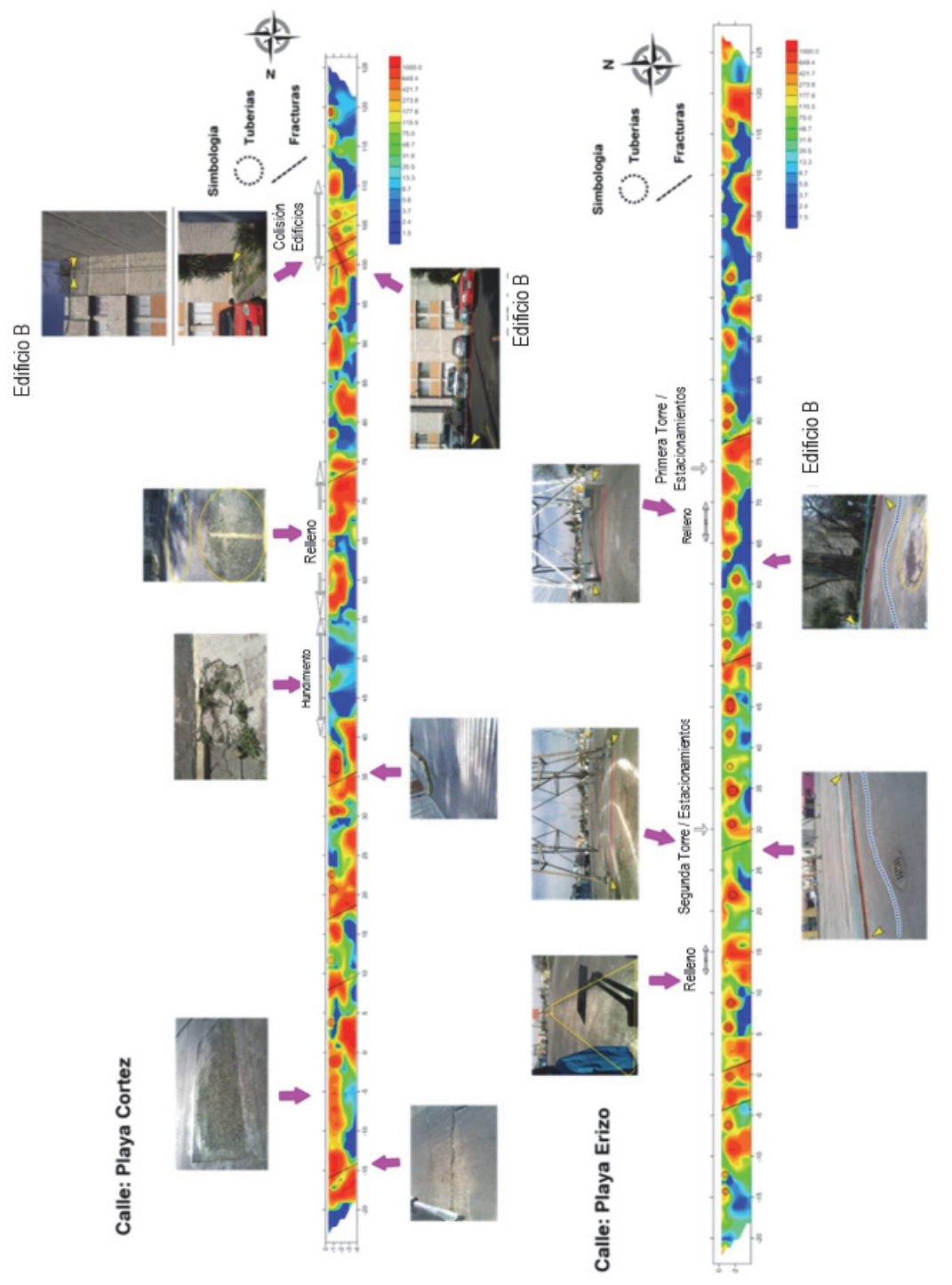

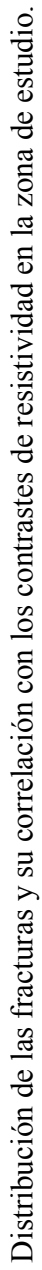

$\underset{7}{\stackrel{0}{0}}$ 


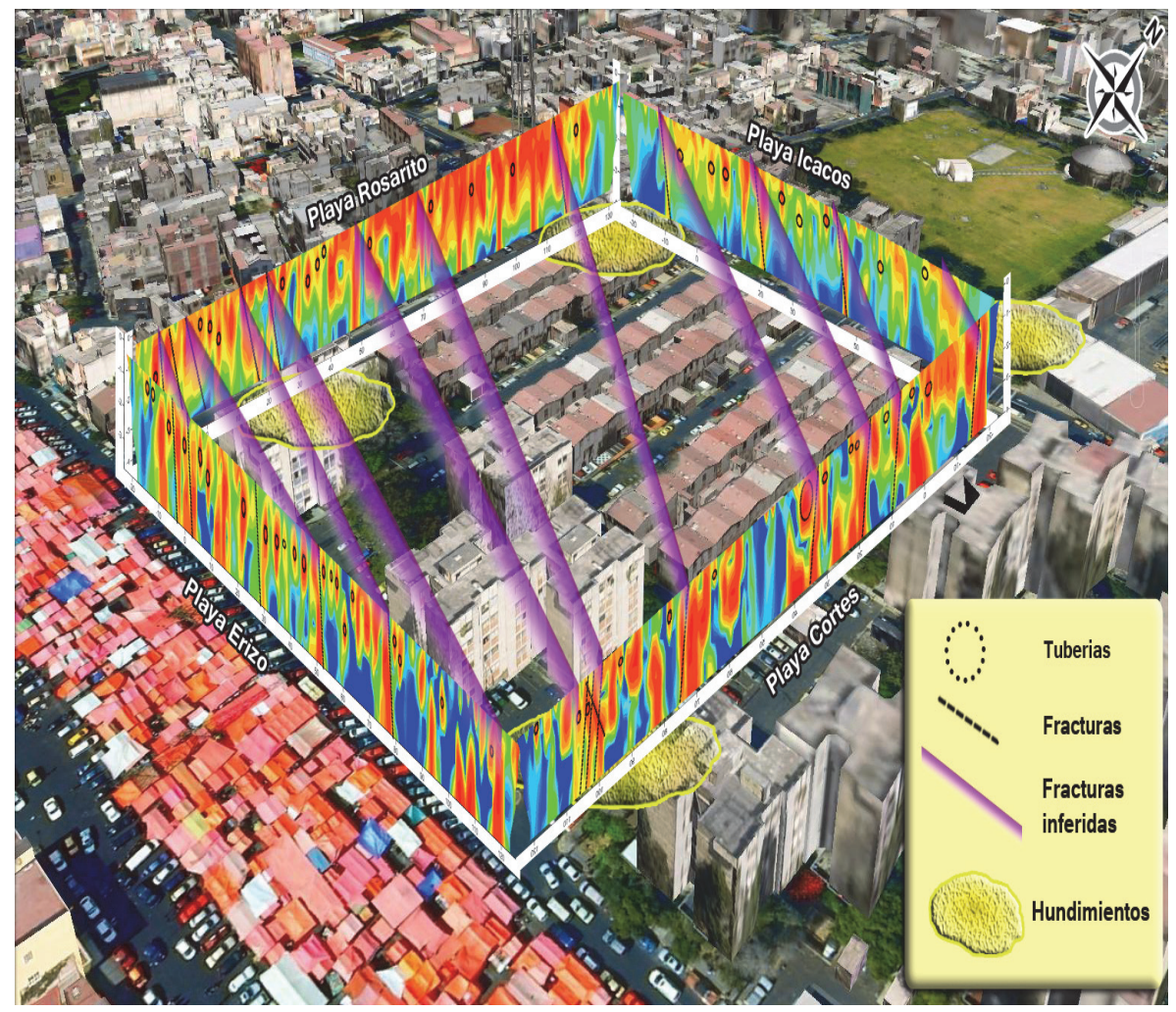

Figura 7. Localización de los perfiles en la zona de casas, correspondiente al barrio de Santiago. En general, la dirección de las principales fracturas inferidas muestra una dirección preferencial NW-SE. Los hundimientos o zonas de subsidencia coinciden en áreas en donde se ubican edificios que pueden estar asociados al gran peso que representa cada uno de ellos, y que provoca colisiones en sus estructuras y un creciente riesgo a sus residentes.

Tras la interpretación de los cuatro perfiles, se puede correlacionar el perfil 1 y 4 donde se logró apreciar que la abertura entre los edificios es debido, muy probablemente, a que existe una alta saturación de agua, que se observa en la mayor parte de los perfiles (bajos valores de resistividad, $>10 \mathrm{Ohm}-\mathrm{m}$ ). Las arcillas que soportan a esta estructura poseen una alta concentración de agua que provoca una fuerte inestabilidad estructural, produciendo las grietas observadas en esta construcción. Por lo que es posible que la cimentación de estos edificios posea filtraciones, provocando la inestabilidad de los mismos. 


\section{Barrio de Santiago}

Esta fue la segunda zona de estudio que pertenece en la porción centro suroeste de la delegación. Se encuentra a las orillas del canal de la Viga, que existía desde tiempos prehispánicos. En esta zona se llevaron a cabo cuatro perfiles de forma tal que pudieran rodear las construcciones que mostraron daño en su estructura. La longitud total de estos perfiles fue de 555 metros (Figura 7). Tras el procesamiento de los datos obtenidos en la zona habitacional del Barrio de Santiago, se obtuvieron cuatro modelos.

Al unir los cuatro perfiles del Barrio de Santiago se logra obtener una vista completa, que nos muestra una imagen más detallada y abre el panorama para comprender con mejor precisión lo que ocurre en el subsuelo así como la dirección preferencial de las fracturas inferidas. Para estimar la dirección de las fracturas mostradas, se tomó en cuenta la visualización superficial de las fracturas en campo, documentadas en imágenes tomadas in situ y colocadas en los puntos que corresponden en cada perfil.

Es importante mencionar que la estructuración civil pudiese estar conformada por cimentaciones superficiales o lozas de cimentación, dichas construcciones son aquellas que están destinadas a soportar el peso de la estructura que estará sobre ellas, así como distribuir las cargas de la edificacion en un plano de apoyo horizontal, planteando un complejo problema de interacción y compatibilidad entre el terreno y la estructura, casi siempre se construyen a poca profundidad bajo la superficie, esto garantiza que la aplicación de estas cargas no provoque en el suelo asentamientos o hundimientos, que puedan ocasionar daños a la casa y a que el suelo no presente fallas por exceder la resistencia o capacidad de carga.

Sin embargo sabemos que esto no se cumplió en las zonas de estudio, notamos la gran diferencia entre dos tipos principales de estructuras, la primera de casa habitación y la segunda de edificios, también percibimos que la forma y el peso original con las que fueron diseñadas las casas se encuentran alteradas, casas que solo contemplaban un piso ahora tienen dos o más, sumado a esto la cercanía con la zona de edificios que cuentan con cinco pisos cada uno, teniendo zonas sin peso aparente como estacionamientos y áreas verdes, esto nos permite concluir que se tiene una mala distribución de peso.

Debido a lo anterior podemos notar que las fracturas más notables se localizaron en el área de estacionamientos de la unidad habitacional, contraria a la zona de casas-habitación; esto es debido a que representan comparativamente menor peso, por lo tanto no se lograron ver fracturas evidentes en la superficie, aunque esto no indique que no existan fracturas en el subsuelo que atraviesen esa zona a profundidad. De esta forma podemos inferir que en las imágenes de resistividad las fracturas pueden pertenecer a la zona de losa de cimentación, que no es muy profunda, de acuerdo al peso y el tipo de subsuelo. 
Las características del terreno y el seguimiento visual en superficie sugieren que las anomalías de alta resistividad observadas se deben a los progresivos rellenos de asfalto, y a la presencia de atarjeas, tuberías de agua y de drenaje que se encuentran en el lugar; y las bajas resistividades se podrían asociar a zonas de saturación en el subsuelo. Esto ha provocado hundimientos y fracturamientos visibles a lo largo de las calles y avenidas que rodean la zona habitacional. En general, las principales fracturas inferidas muestran una dirección preferencial NW-SE. Los hundimientos o zonas de subsidencia coinciden en áreas en donde se ubican edificios que pueden estar asociados al gran peso que representa cada uno de ellos, y que provoca colisiones en sus estructuras y un creciente riesgo a sus residentes.

\section{Tratamiento de los datos}

Sabemos que los datos obtenidos mediante el método capacitivo, están afectados por ruido, cuyo origen en su mayoría es producido por variaciones muy superficiales de la resistividad. Este efecto puede alterar nuestra percepción de anomalías en el perfil, por esta razón se recurrió a eliminar estas variaciones de manera manual, para controlar la manera en que se suavizan o eliminan los datos ruidosos. En los datos de la línea 2, se eliminaron manualmente los picos o los valores fuera de rango, realizando una comparación entre los valores de cada receptor.

Mediante el software comercial Earth Imager 2D del Advance Geosciences Inc. (AGI, 2009) los datos se invirtieron obteniendo los resultados mostrados en las figuras correspondientes.

\section{Conclusiones}

El método capacitivo en el que está basado el equipo geofísico utilizado (OhmMapper) funcionó para poder observar las fracturas y anomalías en la parte más superficial del subsuelo llegando así a profundidades no mayores de 5 metros. Lo anterior produce una buena resolución. Este método es auxiliar y brinda un buen reconocimiento de la zona de manera general, puede ser de gran ayuda para otros estudios, utilizando diversas tecnologías. El trabajo muestra que se lograron visualizar fracturas en los perfiles, que coinciden con los hundimientos y daños en las estructuras de los edificios de ambas zonas de estudio.

El tratamiento manual realizado a los datos de los perfiles, ayudaron a visualizar el cambio en los datos. Esto puede ser muy significativo en la representación de las anomalías y se debe tener cuidado con la edición de los mismos al momento de eliminarlos de manera automática. El uso indiscriminado de este tipo de programas podría hacer que mucha información útil se perdiera, lo que resultaría en una con- 
cepción errónea de los datos, debido a esto es recomendable analizar los datos crudos arrojados por el equipo antes de procesarlos.

El análisis de los perfiles muestra un sistema de fracturas inferidas que siguen una dirección preferencial NW-SE en ambas zonas de estudio, lo anterior podría tener relación con estudios anteriores que se han desarrollado en zonas cercanas (Zamudio-Angeles et al., 2010) y (Aguilar, 2012). Los resultados obtenidos con el equipo Ohm-Mapper fueron suficientes para determinar la existencia de fracturas así como la posibilidad de inferir su dirección y relación con los daños visibles observados en la superficie.

La parte correspondiente a la remediación del problema queda en manos de las autoridades correspondientes o ejecutoras para el monitoreo apropiado del fenómeno; de esta forma tratar de evitar un riesgo mayor a los habitantes.

\section{Referencias}

AGI, 2009. Instruction Manual for EarthImager 2D version 1.5.0 Resistivity Inversion Software, Austin, Texas

Aguilar, Diego A., 2012. Detección de fracturamiento y subsidencia en la unidad habitacional la concordia de la delegación Iztacalco mediante tomografía eléctrica 3D, tesis de licenciatura, Facultad de Ingeniería, UNAM, México, 89 pp.

Mooser, F., 1956. "Los ciclos del vulcanismo que forman la Cuenca de México". Congreso Geol. Internacional 20. Simposio sobre vulcanología del Cenozoico, 2, 337-348

Mooser, F., 1967. "Bosquejo geológico del extremo sur de la Cuenca de México". Congreso Geol. Internacional 20, Libro-guía de la Excursión C-9, 9-16.

Mooser, F., 1975. Memorias de Obras del sistema de Drenaje Profundo del Distrito Federal, Secciones Geológicas.

Orellana, S.E., 1982. Prospección geoeléctrica en corriente continua, vol. 1, Paraninfo 2a. ed., Madrid, 578 pp.

René E. Chávez, Gerardo Cifuentes-Nava, Andrés Tejero, J. Esteban HernándezQuintero and Diana Vargas, 2014. Spatial 3D electric resistivity tomography (ERT) array applied to detect buried fractures in urbana reas: San Antonio Tecomitl, Milpa Alta, Mexico. Geof. Int., 53(4), 425-434.

Trogu, A.; Ranieri, G.; Fischanger F., 2011. 3D electrical resistivity tomography to improve the knowledge of subsoil below existing buildings. Enviromental Semeiotics, 4(4), 63-70.

Zamudio-Ángeles, D.J.; Marín-Córdova; S. y Cambrón, R.J.F., 2010. Estudio geológico estratigráfico para definir zonas de riesgo en cinco localidades de la delegación Iztacalco. Gobierno del Distrito Federal, Delegación Iztacalco. Reporte reservado al Gobierno de la Ciudad de México. 